\title{
Topical Issue on Branching Dynamics at the Mesoscopic Scale
}

\author{
Published online: 29 September 2020 - C EDP Sciences / Società Italiana di Fisica / Springer-Verlag GmbH \\ Germany, part of Springer Nature, 2020
}

\begin{abstract}
Branching structures are commonly observed in nature: from plants to river networks, from snowflakes to flashing lightning. At mesoscopic scale, branching dynamics has also long been of fundamental interest in numerical studies of scientific and engineering significance in materials science: from cracking on the surface to snowflake formation in the air, from Saffman-Taylor instability in Hele-Shaw cells to dendritic growth in the solidification of alloys. Branching phenomena originate from interfacial instability and evolve into complex spatio-temporal patterns at conditions far from equilibrium. Although the basic governing equations for branching dynamics in simple systems are well known, the coupling of heat and mass transport at macroscale and the anisotropic effects of surface tension and kinetics at microscopic scale produces a rich of variety of branching patterns. Branching dynamics at mesoscopic scale, which bridges system conditions (macro) and structure evolution (micro), is the key to understanding the essential nonlinear physics and possible universality of branching structures. Despite the long history of research on branching structures, we still do not fully understand branching dynamics, due to the complexity and diversity of pattern formation and
\end{abstract} selection.

This Topical Issue highlights both experimental and numerical methods that can enrich our understanding of the underlying physics of branching dynamics at mesoscopic scale. Some contributions are devoted to answering the following questions: To what extent does branching dynamics depend on the local composition-dependent interfacial anisotropy and external field? How are the competition and cooperation between grains affected by different orientations? How do branched cracking patterns form under external fields? How do complex growth patterns form in a variety of conditions? How do branched dendrimers form in aqueous solution? The questions addressed by these contributions are important in both fundamental physics and industrial engineering.

This Topical Issue illustrates how branching dynamics depends on edge energy [1] and local composition-dependent interfacial anisotropy [2], thermoelectric effects under external electric field [3], and the effect of gravity [4]. The contributions are devoted to branching arms fragmentation, coarsening [5] and simultaneous melting [6] as well as peritectic transformation [7]. The orientation dependence of branching dynamics is studied in 2-dimensional [8,9] and full 3dimensional systems [10]. Complex branched growth patterns result in complex competitive growth modes. Competitive dendrite growth [11] and seaweed growth [12] are investigated by using numerical simulations and experiments. Further contributions are devoted to branched cracking patterns in carbon nanotube layers at the air-water interface [13] as well as branched cracking patterns in layers of Laponite dried under external fields [14]. The closing contribution explores the formation of amidoamine dendrimers in aqueous solution [15]. Office.

We are delighted to offer our readers this special issue and wish to express our sincere thanks to the Editorial 


\section{References}

1. S. Fashu et al., Eur. Phys. J. E 43, 57 (2020).

2. L. Wang, L. Yang, Eur. Phys. J. E 43, 48 (2020).

3. L. Fu et al., Eur. Phys. J. E 43, 45 (2020).

4. J. Wu et al., Eur. Phys. J. E 43, 30 (2020).

5. H. Fang et al., Eur. Phys. J. E 43, 44 (2020).

6. C. Xiang et al., Eur. Phys. J. E 43, 5 (2020).

7. Y. Fan et al., Eur. Phys. J. E 43, 17 (2020).

8. Y. Wang et al., Eur. Phys. J. E 43, 41 (2020).

9. S. Chu et al., Eur. Phys. J. E 43, 35 (2020).

10. X. Dong et al., Eur. Phys. J. E 43, 28 (2020).

11. M. Hu et al., Eur. Phys. J. E 43, 16 (2020).

12. K. Ankit, M. Glicksman, Eur. Phys. J. E 43, 14 (2020).

13. Y. Zhang et al., Eur. Phys. J. E 42, 105 (2019).

14. A. Ghosh et al., Eur. Phys. J. E 43, 33 (2020).

15. J. Liu et al., Eur. Phys. J. E 43, 7 (2020). 